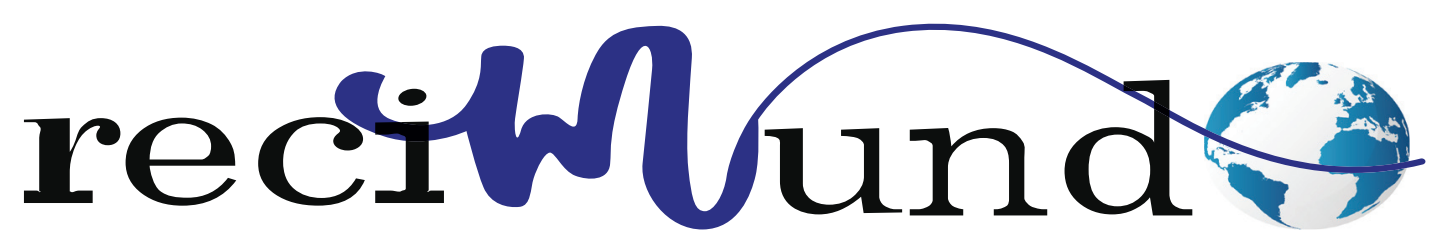

Revista Científica Mundo de la Investigación y el Conocimiento

DOI: $10.26820 /$ recimundo/4.(2).mayo.2020.40-52

URL: http://recimundo.com/index.php/es/article/view/821

EDITORIAL: Saberes del Conocimiento

REVISTA: RECIMUNDO

ISSN: 2588-073X

TIPO DE INVESTIGACióN: Artículo de Revisión

Código UNESCO: 32 Ciencias Médicas; 3201 Ciencias Clínicas

PAGINAS: 40-52

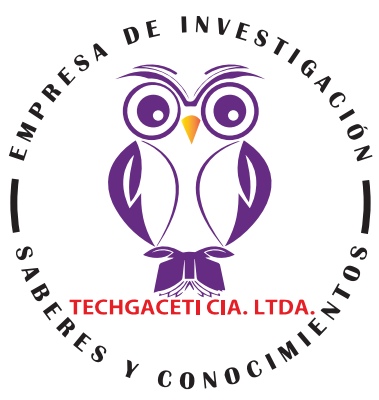

\title{
Manejo del dolor en el paciente con ventilación mecánica en el
} servicio de urgencias

Pain management in the patient with mechanical ventilation in the emergency department

Manejo da dor no paciente com ventilação mecânica no pronto-socorro

Andrés Fernando Alcocer Ortega'; Ángel Fabian Cando Llangari2; Diego Hernan Yambay Lemache3;

Dilmar Alexis Taipe Cofre ${ }^{4}$

\section{RECIBIDO: 15/03/2020 ACEPTADO: 20/03/2020 PUBLICADO: 20/05/2020}

1. Médico; Investigador Independiente; Quito, Ecuador; af_andres-fernandomed@hotmail.com; (D) https://orcid. org/0000-0003-4172-6601

2. Médico General; Investigador Independiente; Riobamba, Ecuador; fabiancl|@hotmail.es; (D) https://orcid.org/0000-0001-8640-808X

3. Médico General; Investigador Independiente; Riobamba, Ecuador; diegoyambay-87@hotmail.es; (D) https:// orcid.org/0000-0003-1406-6249

4. Médico; Investigador Independiente; Quito, Ecuador; dilmar.alexist@gmail.com; (D) https://orcid.org/00000003-2686-3421

\section{CORRESPONDENCIA}

Andrés Fernando Alcocer Ortega

af_andres-fernandomed@hotmail.com

\section{Quito, Ecuador}




\section{RESUMEN}

La movilización temprana de pacientes críticos con ventilación mecánica es una práctica de fisioterapia avanzada. Requiere conocimiento y habilidades especializadas en áreas específicas como la anatomía y la fisiopatología cardiovascular y pulmonar, la ventilación mecánica y la evaluación del sistema de suministro de oxígeno a través de la monitorización hemodinámica. El conocimiento y las habilidades integrales en estas áreas afectarán el proceso de toma de decisiones clínicas y la prescripción del tratamiento para pacientes tan complejos. La implementación de un programa temprano de fisioterapia para pacientes seleccionados en ventilación mecánica con un enfoque en movilidad temprana y caminar es esencial para minimizar el deterioro funcional. El uso de un enfoque interdisciplinario es imprescindible para obtener resultados exitosos. Los fisioterapeutas son un miembro integral del equipo interdisciplinario que atiende a un paciente en la Unidad de Cuidados Intensivos (UCI) y están calificados de manera única para diseñar e implementar planes de tratamiento para mejorar la función. Los fisioterapeutas deben ser competentes, seguros y utilizar habilidades de pensamiento clínico sólidas cuando se enfrentan a los desafíos de la atención al paciente en la UCl. Este artículo incluye la justificación de un programa temprano de movilidad y marcha, el conocimiento requerido para un tratamiento seguro y efectivo, y los enfoques de tratamiento que optimizarán la función de estos pacientes.

Palabras clave: Ventilación mecánica, Manejo del dolor, Fisioterapia.

\section{ABSTRACT}

Early mobilization of critically ill patients with mechanical ventilation is an advanced physical therapy practice. It requires specialized knowledge and skills in specific areas such as cardiovascular and pulmonary anatomy and pathophysiology, mechanical ventilation, and evaluation of the oxygen delivery system through hemodynamic monitoring. Comprehensive knowledge and skills in these areas will affect the clinical decision-making process and treatment prescription for such complex patients. Implementation of an early physical therapy program for selected mechanical ventilation patients with a focus on early mobility and walking is essential to minimize functional decline. The use of an interdisciplinary approach is essential to obtain successful results. Physical therapists are an integral member of the interdisciplinary team caring for a patient in the Intensive Care Unit (ICU) and are uniquely qualified to design and implement treatment plans to improve function. Physiotherapists must be competent, confident, and use sound clinical thinking skills when faced with the challenges of patient care in the ICU. This article includes the rationale for an early mobility and gait program, the knowledge required for safe and effective treatment, and treatment approaches that will optimize the function of these patients.

Keywords: Mechanical ventilation, Pain management, Physiotherapy.

\section{RESUMO}

A mobilização precoce de pacientes críticos com ventilação mecânica é uma prática fisioterapêutica avançada. Requer conhecimentos e habilidades especializadas em áreas específicas, como anatomia e fisiopatologia cardiovascular e pulmonar, ventilação mecânica e avaliação do sistema de fornecimento de oxigênio por meio do monitoramento hemodinâmico. O conhecimento e as habilidades abrangentes nessas áreas afetarão o processo de tomada de decisão clínica e a prescrição do tratamento para pacientes tão complexos. A implementação de um programa de fisioterapia precoce para pacientes selecionados com ventilação mecânica, com foco na mobilidade e na marcha precoces, é essencial para minimizar o declínio funcional. O uso de uma abordagem interdisciplinar é essencial para obter resultados bem-sucedidos. Os fisioterapeutas são um membro integrante da equipe interdisciplinar que cuida de um paciente na Unidade de Terapia Intensiva (UTI) e são qualificados de maneira única para projetar e implementar planos de tratamento para melhorar a função. Os fisioterapeutas devem ser competentes, confiantes e usar boas habilidades de pensamento clínico quando confrontados com os desafios do atendimento ao paciente na UTI. Este artigo inclui a justificativa para um programa precoce de mobilidade e marcha, o conhecimento necessário para um tratamento seguro e eficaz e abordagens de tratamento que otimizarão a função desses pacientes.

Palavras-chave: Ventilação mecânica, Manejo da dor, Fisioterapia. 


\section{Introducción}

La fisioterapia se proporciona con el objetivo de mejorar el rendimiento funcional, prevenir los efectos del descondicionamiento musculoesquelético y lograr una calidad de vida óptima para un individuo con disfunción del movimiento. La implementación de un programa de fisioterapia en pacientes con deterioro funcional es ampliamente aceptada. Sin embargo, los pacientes con ventilación mecánica en la unidad de cuidados intensivos (UCI) son desatendidos por los fisioterapeutas. La configuración de la UCI en sí mismo promueve la movilidad limitada debido a la presencia intimidante y abrumadora de múltiples líneas y archivos adjuntos. El problema se agrava cuando el paciente requiere soporte ventilatorio (Guide to Physical Therapist Practice, 2011). La intubación y la ventilación mecánica generalmente se perciben como barreras para la colocación y movilización del cuerpo. Además, los pacientes que requieren ventilación mecánica a menudo presentan múltiples comorbilidades, lo que podría evitar que se desconecten del ventilador.

Este complejo entorno, donde los pacientes en la $\mathrm{UCl}$ requieren ventilación mecánica prolongada, presenta desafíos únicos para los fisioterapeutas. No se ha desarrollado un consenso entre los expertos para lo que define la ventilación mecánica prolongada, por lo tanto, el rango reportado en la literatura varía desde tan solo 2 días hasta 30 días o más. El aumento de la duración de la estadía (LOS) en una UCI se suma a un alto costo de la atención, alta morbilidad y mortalidad y una disminución de la calidad de vida (Douglas, Daly, Gordon, \& Brennan, 2012). Todos estos resultados negativos son los principales factores que contribuyen al mal estado funcional, ya que estos pacientes sufren una gran pérdida de la función fisiológica. La implementación de un programa temprano de fisioterapia para pacientes seleccionados en ventilación mecánica con un enfoque en la movilidad temprana y ca- minar en la $\mathrm{UCl}$ es esencial para minimizar el deterioro funcional.

Aunque se recomienda encarecidamente la movilización temprana, no existe evidencia clara y establecida de que ayude a los resultados deseados, por ejemplo, reduzca la estancia hospitalaria, mejore la morbilidad y la mortalidad y mejore la calidad de vida del paciente y la familia, los fisioterapeutas son miembros integrales del equipo interdisciplinario que atiende a un paciente en la $\mathrm{UCI}$ (Stiller, 2015). Los fisioterapeutas están especialmente calificados para diseñar e implementar planes de tratamiento cuando los resultados se dirigen hacia la mejora de la función. Sin embargo, aún no se han establecido estándares nacionales y mundiales para la prestación de servicios de fisioterapia a pacientes en $\mathrm{UCl}$, esto el artículo se centrará en los aspectos clínicos de la movilidad temprana y la marcha de los pacientes con ventilación mecánica para mejorar o eliminar el dolor.

\section{Metodología}

Para el desarrollo de este proceso investigativo, se plantea como metodología la encaminada hacia una orientación científica particular que se encuentra determinada por la necesidad de indagar en forma precisa y coherente una situación, en tal sentido (Davila, 2015) define la metodología "como aquellos pasos previos que son seleccionados por el investigador para lograr resultados favorables que le ayuden a plantear nuevas ideas". (p.66)

Lo citado por el autor, lleva a entender que el desarrollo de la acción investigativa busca simplemente coordinar acciones enmarcadas en una revisión bibliográfica con el fin de complementar ideas previas relacionadas al manejo del dolor en pacientes con respiración mecanica a través de una revisión de literatura, para así finalmente elaborar un cuerpo de consideraciones generales que ayuden a ampliar el interés propuesto. 


\section{Tipo de Investigación}

Dentro de toda práctica investigativa, se precisan acciones de carácter metodológico mediante las cuales, se logra conocer y proyectar los eventos posibles que la determinan, así como las características que hacen del acto científico un proceso interactivo ajustado a una realidad posible de ser interpretada. En este sentido, se puede decir, que la presente investigación corresponde al tipo documental, definido por Castro (2016), "se ocupa del estudio de problemas planteados a nivel teórico, la información requerida para abordarlos se encuentra básicamente en materiales impresos, audiovisuales y /o electrónicos". (p.41).

En consideración a esta definición, la orientación metodológica permitió la oportunidad de cumplir con una serie de actividades inherentes a la revisión y lectura de diversos documentos donde se encontraron ideas explicitas relacionadas con los tópicos encargados de identificar a cada característica insertada en el estudio. Por lo tanto, se realizaron continuas interpretaciones con el claro propósito de revisar aquellas apreciaciones o investigaciones propuestas por diferentes investigadores relacionadas con el tema de interés, para luego dar la respectiva argumentación a los planteamientos, en función a las necesidades encontradas en la indagación.

\section{Fuentes Documentales}

El análisis correspondiente a las características que predomina en el tema seleccionado, llevan a incluir diferentes fuentes documentales encargadas de darle el respectivo apoyo y en ese sentido cumplir con la valoración de los hechos a fin de generar nuevos criterios que sirven de referencia a otros procesos investigativos. Para (CASTRO, 2016) las fuentes documentales incorporadas en la investigación documental o bibliográfica, "representa la suma de materiales sistemáticos que son revisados en forma rigurosa y profunda para llegar a un análisis del fenómeno".(p.41). Por lo tanto, se procedió a cumplir con la realización de una lectura previa determinada para encontrar aquellos aspectos estrechamente vinculados con el tema, con el fin de explicar mediante un desarrollo las respectivas apreciaciones generales de importancia.

\section{Técnicas para la Recolección de la Infor- mación}

La conducción de la investigación para ser realizada en función a las particularidades que determinan a los estudios documentales, tiene como fin el desarrollo de un conjunto de acciones encargadas de llevar a la selección de técnicas estrechamente vinculadas con las características del estudio. En tal sentido, (Bolívar, 2015), refiere, que es "una técnica particular para aportar ayuda a los procedimientos de selección de las ideas primarias y secundarias". (p. 71).

Por ello, se procedió a la utilización del subrayado, resúmenes, fichaje, como parte básica para la revisión y selección de los documentos que presentan el contenido teórico. Es decir, que mediante la aplicación de estas técnicas se pudo llegar a recoger informaciones en cuanto a la revisión bibliográfica de los diversos elementos encargados de orientar el proceso de investigación. Tal como lo expresa, (Bolívar, 2015) "las técnicas documentales proporcionan las herramientas esenciales y determinantes para responder a los objetivos formulados y llegar a resultados efectivos" (p. 58). Es decir, para responder con eficiencia a las necesidades investigativas, se introdujeron como técnica de recolección el método inductivo, que hizo posible llevar a cabo una valoración de los hechos de forma particular para llegar a la explicación desde una visión general.

Asimismo, se emplearon las técnicas de análisis de información para la realización de la investigación que fue ejecutada bajo

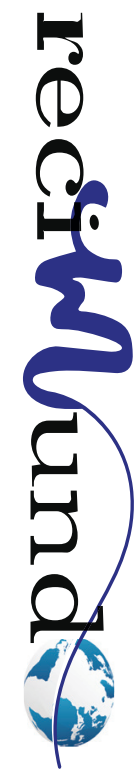


la dinámica de aplicar diversos elementos encargados de determinar el camino a recorrer por el estudio, según, (Bolívar, 2015) las técnicas de procesamiento de datos en los estudios documentales "son las encargadas de ofrecer al investigador la visión o pasos que debe cumplir durante su ejercicio, cada una de ellas debe estar en correspondencia con el nivel a emplear" (p. 123). Esto indica, que para llevar a cabo el procesamiento de los datos obtenidos una vez aplicado las técnicas seleccionadas, tales como: fichas de resumen, textual, registros descriptivos entre otros, los mismos se deben ajustar al nivel que ha sido seleccionado.

\section{Resultados}

\section{Conocimientos necesarios para trabajar con pacientes en ventilación mecánica en la UCI}

La movilización temprana de pacientes críticos con ventilación mecánica es una práctica de fisioterapia avanzada. Requiere educación y habilidades especializadas en áreas específicas que afectan el proceso clínico y de toma de decisiones, así como la prescripción del tratamiento para pacientes tan complejos. El desarrollo de esta experiencia le permitirá al terapeuta comunicarse con confianza con médicos, enfermeras, terapeutas respiratorios y cualquier otro clínico. Para proporcionar una práctica adecuada y segura, se espera que los fisioterapeutas que trabajan en la $\mathrm{UCl}$ tengan una sólida base de conocimiento de lo siguiente:

- Anatomía y fisiología cardiovascular y pultonaria.

- Fisiopatología de los trastornos cardiovasculares y pulmonares y el efecto sobre el transporte y el suministro de oxígeno.

- Valoración cardiovascular y pulmonar

- Conocimiento y capacidad para identificar ritmos cardíacos anormales.
- Sistemas de monitoreo y soporte vital utilizados habitualmente en la UCl

- Ventilación mecánica

- Comprensión exhaustiva de las vías respiratorias artificiales.

- Cuidado respiratorio y equipo suplementario de suministro de oxígeno.

- Medicamentos de uso común en la UCI y sus implicaciones para la práctica de fisioterapia.

- Conocimiento e interpretación de los valores de laboratorio.

- El papel de cada miembro del equipo interdisciplinario de la UCI

- Familiaridad con los procedimientos y políticas de emergencia en la UCI donde practica

\section{Terminología de ventilador}

\section{A / C: CONTROL DE ASISTENCIA}

- Modo sin destete

- La frecuencia y el volumen corriente se configuran para proporcionar un mínimo de ventilación minuto

- El paciente puede generar tantas respiraciones como sea necesario activando el ventilador

- En cada esfuerzo respiratorio espontáneo generado por el paciente, la máquina administra el volumen corriente preestablecido

- Los pacientes generalmente toleran mayores demandas durante la fisioterapia si son médicamente estables

\section{SIMV: VENTILACIÓN OBLIGATORIA IN- TERMITENTE SINCRONIZADA}

- Modo de destete

- La frecuencia y el volumen corriente se configuran para proporcionar un mínimo de ventilación minuto. El paciente puede respirar espontáneamente entre respiraciones del ventilador.

- En cada respiración espontánea, el paciente recibirá tanto volumen como pueda generar 
- A menudo se usa junto con PSV

\section{CPAP: PRESIÓN POSITIVA CONTINUA DE LA VÍA AÉREA}

- Modo de ventilación espontánea

- Modo de destete

- CPAP mantiene presión positiva continuamente en las vías respiratorias

- Se agrega soporte de presión para aumentar el volumen corriente del paciente

\section{PD: VENTILACIÓN DE APOYO A PRESIÓN}

- Se aplica solo a respiraciones espontáneas

- Se puede aplicar a la respiración espontánea de un paciente durante SIMV o CPAP

- Una vez que el paciente activa el ventilador, se entrega la presión positiva preestablecida

- El volumen no está preestablecido, el soporte de presión aumenta el volumen corriente

- El paciente controla la frecuencia respiratoria y el tiempo inspiratorio.

\section{NIPPV: VENTILACIÓN DE PRESIÓN POSI- TIVA NO INVASIVA}

- Utiliza una máscara en lugar de una vía aérea artificial.

- Se utiliza cuando se espera ventilación a corto plazo para la exacerbación de la EPOC, la extubación fallida, la neumonía, la insuficiencia cardíaca congestiva, el edema pulmonar

\section{PEEP: PRESIÓN POSITIVA DE EXPIRA- CIÓN FINAL}

- Presión restante en el circuito del ventilador al final de la caducidad

- PEEP aumenta la capacidad residual funcional

- Aumenta el volumen pulmonar para prevenir o corregir la atelectasia

- Aumenta la presión media de las vías respiratorias para mejorar la oxigenación

- Evita el colapso de las vías respiratorias

\section{FRACCIÓN DE OXÍGENO INSPIRADO}

- Oxígeno suplementario administrado por el ventilador expresado como porcentaje

\section{Proceso de toma de decisiones}

Una de las habilidades más impotentes de un fisioterapeuta que trabaja en una $\mathrm{UCl}$ es la capacidad de tomar decisiones clínicas sobre cuándo iniciar, retrasar, continuar o detener actividades para pacientes críticos. El clínico debe procesar grandes cantidades de información en poco tiempo para tomar las decisiones apropiadas. El programa de fisioterapia debe basarse en la condición médica y los objetivos del paciente, no en el equipo de la UCl y la presencia de ventilación mecánica. El conocimiento y la comprensión limitados del equipo de la $\mathrm{UCl}$ y la ventilación mecánica limitarán significativamente los resultados para estos pacientes (Holtackers \& Irwin, 1995).

El proceso de toma de decisiones comienza cuando el clínico llega al lado de la cama y comienza reuniendo la información relevante. Luego, continúa como un proceso dinámico, impulsado por la respuesta, en el que las decisiones clínicas se toman minuto a minuto, en función de una evaluación exhaustiva de la afección médica, los síntomas y la respuesta del paciente a la actividad. Al implementar un proceso impulsado por la respuesta, el terapeuta debe conocer los parámetros establecidos por el equipo médico que maneja al paciente y sus objetivos actuales de atención. Un compromiso fuerte con un enfoque de equipo interdisciplinario es imprescindible para el proceso de toma de decisiones clínicas, así como para la práctica segura. Los fisioterapeutas no necesitan estar preparados para resolver emergencias médicas, sin embargo, deben estar conscientes de los problemas 
médicos inminentes que podrían conducir a una emergencia.

\section{¿Qué pacientes con ventilación mecáni- ca se beneficiarán de un programa tem- prano de movilidad y caminata?}

Se requiere una referencia médica antes de una evaluación de fisioterapia y / o el inicio de cualquier tratamiento. Una vez que se hace una referencia a la fisioterapia, el fisioterapeuta es en última instancia responsable de determinar la idoneidad de un programa temprano de movilidad y caminata para cada paciente. Para fines de fisioterapia, la movilidad temprana significa alentar al paciente a estar activo, erguido y en movimiento una vez que se haya producido la estabilización fisiológica después de la admisión a la UCl.

Algunos criterios para ayudar al clínico en esta determinación incluyen:

- Neurológico: el paciente debe ser capaz de responder a la estimulación verbal y seguir órdenes simples

- Circulatorio: el paciente debe ser hemodinámicamente estable con frecuencia cardíaca, ritmo y presión arterial aceptables sin fármacos vasopresores, por ejemplo, dopamina, vasopresina, norepinefrina

- Respiratorio: el paciente debe tener un patrón ventilatorio adecuado con la frecuencia y el ritmo de la respiración sincronizados con el soporte ventilatorio provisto. El individuo también debe estar recibiendo niveles aceptables de oxígeno suplementario, generalmente menos de $0.6 \mathrm{FiO} 2$, a menos que lo especifique el médico.

\section{Evaluación de fisioterapia}

Una vez que el paciente es referido a un físioterapeuta, se realiza una evaluación integral de fisioterapia. La evaluación ayudará a establecer información de referencia para el paciente y establecer el diagnóstico, el pronóstico y el plan de atención. Es posible que los exámenes deban ser adaptados para la UCI. Muchas pruebas y medidas estándar no son posibles en la UCl y la condición médica del paciente puede limitar el examen preciso. Según los datos recopilados en el examen, los pacientes en $\mathrm{UCl}$ que requieren ventilación mecánica a menudo tienen uno o más de los siguientes impedimentos y limitaciones funcionales:
a. Ventilación deteriorada
b. Intercambio de gas deteriorado
c. Capacidad aeróbica deteriorada
d. Deterioro del rendimiento muscular
e. Incapacidad para realizar movilidad en la cama y transferir actividades de forma independiente
f. Incapacidad para deambular
g. Incapacidad para realizar autocui- dado o ADL instrumentales

\section{Metas de fisioterapia}

Establecer objetivos apropiados para pacientes con ventilación mecánica en la UCI puede ser una tarea difícil para los fisioterapeutas y debe basarse en los resultados previstos. Estos generalmente son los resultados esperados después de que el plan de atención ha sido implementado

\section{Resultados anticipados}

- Se mejora el nivel de excitación

- Función cardiopulmonar optimizada

- Función neuromuscular optimizada

- La pérdida de habilidades funcionales se minimiza.

- Se maximiza la independencia. El proceso de destete se facilita cuando la causa principal de la falla de destete es la debilidad muscular

- Se mejora la actitud del paciente hacia la recuperación.

Las metas de fisioterapia a corto y largo plazo deben ser medibles y limitadas en el 
tiempo. En muchos casos, la condición médica de una paciente mejora rápidamente y el tiempo esperado de ventilación mecánica es corto y predecible. Para tales pacientes, se espera una recuperación funcional a medida que mejora la condición médica. En estos casos, se pueden establecer objetivos orientados a funciones a corto y largo plazo. Establecer objetivos a largo plazo para pacientes más complejos que requieren períodos prolongados de soporte ventilatorio puede no ser razonable. Debido a las fluctuaciones en el estado médico del paciente, así como a un pronóstico impredecible, los objetivos a largo plazo pueden diferirse hasta que el paciente demuestra una mejora en la condición médica.

Las metas siempre deben ser específicas del paciente, basadas en las capacidades individuales y la condición médica general. Las áreas de enfoque para establecer metas para impedimentos y limitaciones funcionales incluyen:

\section{Mejore la fuerza muscular o el control motor para promover una mayor función:}

- Extremidades inferiores

- Extremidades superiores

- Control de cabeza y tronco

\section{Mejora la resistencia:}

- Aumentar las repeticiones de ejercicios para las extremidades superiores e inferiores.

- Aumente el tiempo sentado sin apoyo en el borde de la cama

- Aumente la distancia a pie dentro de los signos y síntomas aceptables

- Agregar actividades aeróbicas como la ergometría y las escaleras de bicicletas y brazos

\section{Mejora progresivamente la movilidad funcional:}

- Actividades de movilidad en la cama
- De pie con dispositivo de asistencia y asistencia

- Traslado a una silla o una cómoda junto a la cama

- Reeducación a pie con un dispositivo de asistencia y asistencia

- Independencia con ADL

\section{EVALUACIÓN DE TERAPIA FÍSICA}

1. Resumen de la tabla: Historial médico pasado y presente

2. Nivel anterior de función: Independencia con la deambulación y las actividades de la vida diaria (ADL), uso de dispositivos de asistencia / equipos de adaptación / equipos médicos duraderos (DME), nivel de actividad, hábitos de ejercicio.

3. Historia social: Recursos familiares y sistema de apoyo, empleo

4. Estado mental: Nivel de conciencia, así como la capacidad de seguir órdenes, presencia de sedación y justificación para su uso, confusión, agitación.

5. Integridad de la piel

6. Estado cardíaco: Frecuencia cardíaca y ritmo, presión arterial, fracción de eyección, presencia de soporte vital invasivo y equipos como catéter de arteria pulmonar, IABP, LVAD, CWHD

7. Estado pulmonar: Configuración del ventilador, valores de gases en sangre arterial, valor de saturación de oxígeno. Resultados de rayos $X$, último cambio de ventilador, proceso de destete y horario

8. Estado neurológico: Presión intracraneal, tono, presencia de parálisis / parecía, coordinación, sensación.

9. Estado musculoesquelético: Fracturas, limitaciones en el rango de movimiento (ROM), presencia de debilidad muscular significativa

10. Medicamentos: especialmente aquellos que pueden afectar potencialmente el rendimiento funcional: corticosteroides, bloqueadores neuromusculares, narcóticos, sedantes

11. Pruebas de laboratorio y diagnóstico relevantes. 
12. Estado funcional actual: Movilidad de la cama, transferencias y capacidad para caminar.

\section{Tratamiento de fisioterapia}

Las intervenciones de fisioterapia en la UCI pueden incluir posicionamiento, drenaje postural, limpieza de las vías respiratorias, reentrenamiento respiratorio, ejercicios terapéuticos, músculo inspiratorio capacitación (IMT), transferencias, reeducación de la marcha y educación del paciente / familia. Como se discutió anteriormente, el enfoque del tratamiento de fisioterapia para pacientes con ventilación mecánica en la UCI debe ser ayudar al paciente a alcanzar la posición erguida, tolerar las actividades de soporte de peso y avanzar hacia la reeducación de la caminata lo antes posible. Las intervenciones de fisioterapia deben seleccionarse con prudencia de una manera orientada a objetivos con enfoque en la función.

La reeducación de la marcha para pacientes que requieren ventilación mecánica en la UCl es el vínculo entre el reposo en cama y la capacidad de soportar peso, caminar y mejorar la movilidad funcional. Se encuentran disponibles sugerencias de dispositivos para ayudar en la deambulación (Smith, Forrest, Evan, \& G., 2006) pero no existe un estándar de atención para guiar la práctica de dichos pacientes. Las actividades con carga de peso pueden ser un gran desafío para los ancianos y la población de pacientes de alta agudeza. La debilidad profunda, la disminución de la resistencia y los múltiples problemas médicos limitan significativamente las ganancias funcionales. El uso de una mesa de inclinación dinámica como complemento de las técnicas de rehabilitación convencionales ha informado en la literatura, permite a los pacientes realizar ejercicios de cadena cerrada en un entorno de gravedad reducida utilizando su propio peso corporal. Algunas veces identificar los pacientes puede ser difícil; sin embar- go, retrasar la movilidad puede poner a los pacientes en riesgo de desarrollar impedimentos secundarios prevenibles y limitaciones funcionales. Algunos pacientes pueden estar listos para moverse antes de que aparezcan o antes de que lo creamos. Una prueba de movilización puede ayudar a determinar si el paciente tiene el control motor y el oxígeno adecuado.

Antes de movilizar a estos pacientes, se deben responder varias preguntas para desarrollar un plan de tratamiento adecuado.

- ¿Cuál es el nivel de participación del paciente?

- ¿Cuál es la gravedad de las deficiencias funcionales del paciente?

- ¿Cuáles son los tratamientos disponibles de elección? ¿Cuáles son las prioridades de tratamiento en este caso? ¿Por qué?

- ¿Cuáles son los parámetros prescriptivos?

- ¿Cuál debería ser la secuencia de tratamiento? ¿Por qué?

- ¿Cuál es el tiempo esperado disponible y el curso del tratamiento?

La buena comunicación con el médico, la enfermera y el terapeuta respiratorio es esencial y deben acordar diariamente la actividad adecuada para cada paciente. La enfermera debe estar disponible para ayudar con las vías intravenosas y el terapeuta respiratorio para ayudar con el manejo del ventilador. Debe existir un plan organizado para garantizar que las líneas y los cables estén conectados adecuadamente para la evaluación y se gestionen durante el tratamiento. Las alarmas a menudo se activan cuando se trabaja con pacientes en una $\mathrm{UCl}$ en un monitor o un ventilador. Los médicos que los signos vitales o la función del ventilador están fuera de los parámetros establecidos por el equipo médico. Cuando suena una alarma, evalúe al paciente. Un paciente alerta y orientado generalmente no está en peligro inmediato. Si un pacien- 
te responde y participa, el tratamiento terapéutico no debe interrumpirse solo por una alarma. Asegúrese de resolver el problema y luego continúe con el tratamiento planificado, si corresponde. Cuando suenan las alarmas:

- Pare el tratamiento momentáneamente

- Observar y brindar apoyo al paciente.

- Determine qué alarma está sonando y por qué

- Tome las medidas adecuadas para resolver los problemas que hacen que suene la alarma.

- Si no está seguro de por qué, o las alarmas no se detienen, llame a la enfermera o al terapeuta respiratorio de inmediato.

- Nunca deje al paciente.

\section{Monitorización del paciente de la $\mathrm{UCl}$ con ventilación mecánica}

El fisioterapeuta debe tener conocimiento de los valores normales de los signos vitales. Se debe tener en cuenta la frecuencia cardíaca (FC), la presión arterial (PA), la saturación de oxígeno (SpO2) y la frecuencia respiratoria $(R R)$ en reposo. En la $U \mathrm{Cl}$, estos signos están disponibles en el monitor de telemetría. El rango aceptable para los signos vitales puede diferir con cada paciente. Determine qué es aceptable para cada paciente antes de cada sesión de terapia una vez que se evalúa a fondo la condición médica general. Los límites para cada signo vital durante la actividad deben ser discutidos y acordados por el equipo interdisciplinario. Debido a la naturaleza crítica de las enfermedades de los pacientes y los cambios constantes en general condición médica, es necesaria una evaluación vigilante para determinar si el paciente está tolerando la intervención planificada. También permite modificar el plan de tratamiento según sea necesario para cumplir con los parámetros establecidos y promover la seguridad. Siempre correlacione los signos vitales con los síntomas y también tenga en cuenta específicamente los cambios repentinos en los signos vitales. Se debe proporcionar soporte ventilatorio y de oxígeno adecuado para que el paciente pueda tolerar mayores niveles de actividad.

\section{Contraindicaciones absolutas para el programa temprano de movilidad y cami- nata}

- Pacientes comatosos que no responden

- Cambios significativos y sintomáticos en el ECG en reposo con compromiso hemodinámico.

- Manejo del paciente en ventilación mecánica en $\mathrm{UCl}$ : programa de movilidad temprana y caminata

- Angina inestable

- Insuficiencia cardíaca no controlada Inestabilidad hemodinámica que requiere dosis altas o múltiples fármacos vasopresores

- Sospecha o conocido aneurisma de disección

- Embolia pulmonar aguda con afección médica inestable.

- Disfunción de oxigenación significativa que requiere altos niveles de oxígeno suplementario, generalmente más de $0.7 \mathrm{FiO} \wedge$, a menos que lo especifique el médico Edema cerebral con ICP incontrolado

- Disfunción neurológica y / o musculoesquelética significativa Fracturas inestables

Barreras para un programa temprano de movilidad y caminata para pacientes con ventilación mecánica en la UCI

Para disminuir la posibilidad de efectos adversos durante el curso de la evaluación y los tratamientos de fisioterapia, se deben evaluar y considerar los siguientes factores antes de establecer un plan de tratamiento:

- Confianza y nivel de experiencia del fisioterapeuta en el entorno de la UCI

- Falta de cooperación del paciente

- Cultura de UCI que promueve el reposo 
en cama

- Edad y nivel de función antes de la admisión a la UCl

- Gravedad de la enfermedad y comorbilidades.

- Reserva cardíaca y pulmonar limitada

- Evaluación limitada de la fuerza, la movilidad y la capacidad de soportar peso.

- Peso corporal del paciente

- Dolor

- Sedación

- Estados nutricionales

- La privación del sueño

- Cooperación interdisciplinaria

- Disponibilidad de personal para ayudar con la movilización

- Número de líneas, monitoreo de tubos y equipos de soporte vital.

\section{Pautas para finalizar una sesión de fisio- terapia en la UCI}

- Dolor intenso en el pecho asociado con cambios en el ECG

- Frecuencia cardíaca superior a la frecuencia cardíaca máxima prevista o límites predeterminados establecidos por el equipo médico

- Hipotensión asociada con mareos, aturdimiento y diaforesis.

- Disnea intolerable asociada con un mayor uso de músculos accesorios, patrón de respiración paradójico, aleteo nasal y cianosis

- Incapaz de mantener una SpO 2 aceptable a pesar del oxígeno suplementario

- Dolor intenso a pesar de la analgesia.

- Fatiga extrema

- El paciente desea parar.

\section{Consejos para el exito}

Debe hacerse algunas preguntas antes de cada evaluación inicial y sesión de tratamiento terapéutico. Las respuestas a estas guiarán su proceso de toma de decisiones clínicas y ayudarán a proporcionar una práctica segura.
- Quien es mi paciente, Información de la revisión del cuadro, enfermera, MD, familia.

- ¿Mi paciente puede participar con la terapia? Despierta, capaz de seguir comandos.

- ¿Es mi paciente hemodinámicamente estable? Mira el monitor, gotas intravenosas.

- ¿Cómo se ve mi paciente? Calma, relajada, incómoda, angustia aguda.

- ¿Cuál es la posición e integridad de las vías respiratorias artificiales, líneas, tubos unidos al paciente?

- ¿Está mi paciente en equipo de soporte vital? Ventilador, bomba de balón intraaórtico (IABP), dispositivo de asistencia ventricular izquierda (LVAD), hemodiálisis venovenosa continua (CWHD). ¿Ha cambiado drásticamente la condición médica de mi paciente en las últimas 24 horas? Cirugía reciente, hipotensión, reintubación ...

- ¿Tengo suficiente ayuda y apoyo del equipo interdisciplinario para garantizar la seguridad del paciente durante la sesión de tratamiento?

Los pacientes con intubación endotraqueal o traqueotomía pueden movilizarse de manera segura cuando se toman las medidas apropiadas (Bailey, Thomsen, Spuhler, \& VJ., 2015) Por lo tanto, los que trabajan los pacientes con ventilación mecánica deben tener un conocimiento profundo de la ventilación mecánica y las vías respiratorias artificiales. Un programa integral de orientación y evaluación de la competencia es la clave para una práctica exitosa de fisioterapia en el entorno de cuidados críticos. El conocimiento y la comprensión limitados del equipo de la UCI pueden limitar los resultados de la terapia para los pacientes de la UCI. Para estar equipado para proporcionar atención segura y efectiva a los pacientes críticos, el fisioterapeuta debe:

- Comprender los principios del transporte de oxígeno y la fisiopatología car- 
diopulmonar básica.

- Comprender la función del equipo de la $\mathrm{UCl}$ y las implicaciones durante las intervenciones terapéuticas.

- Comprender las intervenciones médicas proporcionadas en la UCl

- Trabajar en estrecha colaboración con todos los miembros del equipo de la UCl

- Usa fuertes habilidades de pensamiento crítico

\section{Conclusiones}

Los fisioterapeutas que practican en la $\mathrm{UCl}$ enfrentan desafíos complejos en el cuidado de pacientes con ventilación mecánica. Para muchos fisioterapeutas, la UCI se presenta como un entorno intimidante; sin embargo, nunca deben olvidar su objetivo principal de mejorar la función. Al desarrollar una sólida base de conocimientos y habilidades necesarias para la práctica segura en este entorno clínico, aumentará la confianza de los fisioterapeutas. El uso de un enfoque interdisciplinario es imprescindible para obtener resultados exitosos. Se recomienda el estímulo gravitacional y la marcha de pacientes seleccionados que requieren ventilación mecánica en la $\mathrm{UCl}$, pero no siempre forma parte de la intervención de fisioterapia. Dado que el papel específico del fisioterapeuta en la $\mathrm{UCI}$ no ha sido bien definido, varía considerablemente y el programa de fisioterapia se implementa de acuerdo con la discreción profesional del individuo. Como lo observaron los autores, el concepto de un programa temprano de movilidad y caminata para pacientes seleccionados de la $\mathrm{UCl}$ tiene el potencial de afectar los resultados positivos, que incluyen:

- Prevención de complicaciones secundarias del reposo en cama.

- Alto nivel de satisfacción para enfermeras, pacientes, médicos, terapeutas y familiares.

- Asistencia con el proceso de destete del ventilador a medida que mejoran la fuer- za, la movilidad por flexión y la resistencia

- Disminución de la duración de la estancia hospitalaria y los costos.

Se tiene una necesidad urgente de investigación para proporcionar evidencia científica de que un programa temprano de movilidad y caminata mejora definitivamente los resultados. La profesión de fisioterapia debe establecer las intervenciones óptimas de fisioterapia y determinar su efectividad en el tratamiento de pacientes que requieren ventilación mecánica en la UCI. Esto allanará el camino para que la participación de la fisioterapia sea un estándar de práctica universalmente aceptado en el tratamiento de pacientes médicamente complejos.

\section{Bibliografía}

Bailey, P., Thomsen, G., Spuhler, \& VJ. (2015). Early activity is safe and feasible in respiratory failure patients. Crit Care Med, 139-145.

Bolívar, J. (2015). Investigación Documental. México. Pax.

Castro, J. (2016). Técnicas Documentales. México. Limusa.

Davila, A. (2015). Concepto de terminos cientificos. Caracas: Oasis.

Douglas, S., Daly, B., Gordon, N., \& Brennan, P. (2012). Survival and quality of life: Short term versus long-term ventilator patients. Crit Care Med, 2655-2662.

Guide to Physical Therapist Practice. (2011). Phys Ther, 81:21.

Holtackers, T., \& Irwin, S. (1995). Physical rehabilitation of the ventilator dependent patient. Cardiopulmonary Physical Therapy, 292-304.

Smith, T., Forrest, G., Evan, \& G. (2006). The Albany Medical College Ventilator Walker. Arch Phys Med Rehabil, 1320-1321.

Stiller, K. (2015). Physiotherapy in Intensive Care: Towards an Evidence Based Practice. Chest, 1801-1813. 


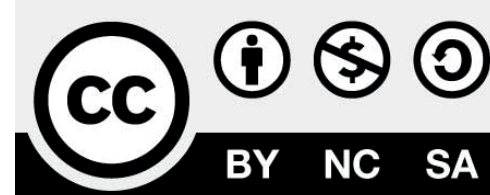

RECONOCIMIENTO-NOCOMERCIAL-COMPARTIRIGUAL CC BY-NC-SA

ESTA LICENCIA PERMITE A OTROS ENTREMEZCLAR, AJUSTARY CONSTRUIR A PARTIR DE SU OBRA CON FINES NO COMERCIALES, SIEMPRE Y CUANDO LE RECONOZCAN LA AUTORÍA Y SUS NUEVAS CREACIONES ESTÉN BAJO UNA LICENCIA CON LOS MISMOS TÉRMINOS.

\section{CITAR ESTE ARTICULO:}

Alcocer Ortega, A., Cando Llangari, Á., Yambay Lemache, D., \& Taipe Cofre, D. (2020). Manejo del dolor en el paciente con ventilación mecánica en el servicio de urgencias. RECIMUNDO, 4(2), 40-52. doi:10.26820/recimundo/4.(2) mayo.2020.40-52 